\title{
Betatron x-ray production in mixed gases
}

\author{
F. Albert ${ }^{a}$, B.B. Pollock ${ }^{a}$, J. Shaw ${ }^{b}$, K.A. Marsh ${ }^{b}$, Y.-H. Chen ${ }^{a}$, D. Alessi ${ }^{a}$, J.E. Ralph ${ }^{a}$, P.A. \\ Michel $^{a}$, A. Pak, ${ }^{a}$, C.E. Clayton ${ }^{b}$, S.H. Glenzer ${ }^{c}$ and C. Joshi ${ }^{b}$ \\ ${ }^{a}$ Lawrence Livermore National Laboratory, NIF and Photon Sciences, 7000 East avenue, \\ Livermore, CA 94550, USA \\ ${ }^{b}$ Department of Electrical Engineering, University of California, Los Angeles, California 90095, \\ USA \\ ${ }^{c}$ SLAC National Accelerator Laboratory, Stanford, California 94309, USA
}

\begin{abstract}
Betatron x-rays with multi-keV photon energies have been observed from a GeV-class laser-plasma accelerator. The experiment was performed using the $200 \mathrm{TW}$ Callisto laser system at LLNL to produce and simultaneously observe GeV-class electron beams and $\mathrm{keV}$ Betatron x-rays. The laser was focused with two different optics (f/8 and $\mathrm{f} / 20$ ), and into various gas cells with sizes ranging from 3 to $10 \mathrm{~mm}$, and containing mixed gases ( $\mathrm{He}, \mathrm{N}, \mathrm{CO} 2, \mathrm{Ar}, \mathrm{Ne}$ ) to accelerate large amounts of charge in the ionization induced trapping regime. $\mathrm{KeV}$ betatron x-rays were observed for various concentrations of gases. Electron spectra were measured on large image plates with the two-screen method after being deflected by a large 0.42 Tesla magnet spectrometer. Betatron oscillations observed on the electron spectra can be benchmarked against a simple analytical model (RungeKutta algorithm solving the equation of motion of an electron in the wakefield), in order to retrieve the electron injection conditions into the wake.
\end{abstract}

Keywords: Betatron x-ray radiation, Laser-wakefield acceleration, laser-plasma interaction.

\section{INTRODUCTION}

Laser Wakefield Acceleration (LWFA) ${ }^{1}$ is one of the most notable applications that resulted from the advent of petawatt-class laser systems. Since the discovery that this mechanism can accelerate monoenergetic electron beams up to energies comparable to those obtained with conventional $\mathrm{rf}$ accelerators ${ }^{2-4}$ but in a table-top setup, novel applications of these electron beams have been constantly increasing. Concurrently, x-ray sources such as synchrotrons, or more recently free-electrons lasers such as the Linac Coherent Light Source (LCLS) ${ }^{5}$ continue to explore new properties of atoms, molecules, condensed matter, warm dense matter or plasmas. In this context, LWFA beams are very attractive to seed the next generation of light sources. Such beams can either be wiggled by an external periodic magnetic structure ${ }^{6}$ or directly by the plasma in the wake of the laser pulse ${ }^{7,8}$ to produce $\mathrm{keV}$ x-rays. The latter example, the betatron x-ray source, first observed in a beam-driven plasma channel ${ }^{9}$ is the subject of this work, and its mechanism can be described as follows: an ultrashort (femtosecond), ultraintense $\left(I>10^{18} \mathrm{~W} / \mathrm{cm}^{-3}\right)$ laser pulse is focused under vacuum on the edge of a gas target. The gas is fully ionized to form a plasma. The laser ponderomotive force (proportional to the gradient of light intensity) plows the electrons of the plasma away from the strong light intensity regions. Because of the very short duration of the laser pulse, the heavier ions stay immobile and a bubble free of electrons is formed in the wake of the pulse. At the back of this bubble, some electrons are trapped, accelerated and wiggled by the electrical fields present in the plasma: these electrons emit the betatron x-rays. This source produces broadband, synchrotron-like radiation in the $\mathrm{keV}$ energy range, ${ }^{10,11}$ within a source size of a few microns, ${ }^{12,13}$ a divergence of less than $100 \mathrm{mrad},{ }^{14}$ and a pulse duration of less than $100 \mathrm{fs} .{ }^{15} \mathrm{X}$-ray phase-contrast imaging is one of the applications of this source showing its potential as a novel x-ray probe. ${ }^{16,17}$ Since betatron x-rays are directly related to the electrons emitting them, the source is also widely used as an electron beam diagnostic. The electron beam emittance and size can be deduced from the x-ray beam profile, ${ }^{14}$ spectrum $^{18}$ or source size,${ }^{19}$ using various x-ray spectroscopy

Further author information: Send correspondence to F.A.

E-mail: albert6@llnl.gov, Telephone: +1925 4226641

Laser Acceleration of Electrons, Protons, and lons II; and Medical Applications of Laser-Generated Beams of Particles II; and Harnessing Relativistic Plasma Waves III, edited by D. A. Jaroszynski, K. W. D. Ledingham, E. Esarey, C. B. Schroeder, W. P. Leemans, Proc. of SPIE Vol. 8779, 87791Q - @ 2013 SPIE · CCC code: 0277-786X/13/\$18 - doi: 
and imaging techniques. Traditionally, betatron x-rays are produced in the blowout regime of laser wakefield acceleration $^{20}$ using pure helium gas, where electrons are self-injected into the wake. This paper investigates the properties of betatron x-ray radiation produced in mixed gases end employs similar electron beam and x-ray characrerization techniques. After a brief review of the electron acceleration scheme in the ionization-induced trapping regime ${ }^{21,22}$ and the betatron x-ray source theory, we review the experimental techniques and results obtained using the 200 TW Callisto laser system at the Jupiter Laser Facility, LLNL.

\section{THEORY AND MODELING}

\subsection{Electron acceleration in the ionization induced trapping regime}

The blowout regime of laser wakefield acceleration has been widely investigated through 3D Particle-In-Cell (PIC) simulations. ${ }^{20}$ In this case, the plasma wavelength $\lambda_{p}$ is superior or equal to the axial laser dimensions $c \tau$, where $\tau$ is the laser pulse length. If the laser intensity is sufficiently high (on the order of $3 \times 10^{18} \mathrm{~W} / \mathrm{cm}^{2}$ for electrons densities of $10^{19} \mathrm{~cm}^{-3}$ ), the laser ponderomotive force expels the plasma electrons toward the low light intensity regions. This leaves an ion cavity (or bubble) in the wake of the laser pulse, with dimensions close to that of the light pulse (several femtoseconds). Electrons injected in this accelerating structure can be accelerated until they outrun the wakefield over a dephasing length $L_{d p}=\frac{2}{3} r \omega_{0}^{2} / \omega_{p}^{2}$ and gain a maximum energy $E_{\max }$ $[\mathrm{GeV}]=0.36(\mathrm{P}[\mathrm{TW}])^{1 / 4} \times\left(L_{d p}[\mathrm{~cm}]\right)^{1 / 2}$, where $\mathrm{P}$ is the peak laser power. The blowout radius $r$ is matched to the laser beam waist $w_{0}: k_{p} r \sim k_{p} w_{0}=2 \sqrt{a_{0}}$. A result of this mechanism is that accelerating high energy electrons is limited either by the dephasing length or the pump depletion length $L_{\text {pump }}=c \tau \omega_{0}^{2} / \omega_{p}^{2}$. One solution is to accelerate the electrons in a capillary discharge to guide the laser pulse and accelerate electrons to $\mathrm{GeV}$ energies. ${ }^{23}$ Since the dephasing length increases with decreasing electron density, operating at lower electron densities is favorable. Unfortunately, for given laser parameters, there is a density threshold below which electrons can no longer be trapped. Ionization-induced trapping ${ }^{21,24}$ has been proposed to permit electron trapping below this threshold to take advantage of large dephasing lengths. Instead of using pure helium gas to drive the wake and accelerate electrons, trace amounts of dopant $\left(\mathrm{N}, \mathrm{CO}_{2}\right)$ are added. For a $\mathrm{He} / \mathrm{N}$ mix, the leading edge of the laser pulse is sufficiently intense to fully ionize the He atoms and strip the outer five electrons of nitrogen. The laser ponderomotive force pushes the electrons out to create the accelerating structure, just like in the case of a pure helium plasma. However, there is a large difference between the ionization potential of the 5th (L-shell) electron and the two K-shell electrons on nitrogen $\left(\mathrm{N}^{6+}\right.$ and $\left.\mathrm{N}^{7+}\right)$. When this step in the ionization potential is matched to the laser intensity profile such that the two nitrogen K-hell electrons are ionized near the peak of the laser electrical field, these electrons are born at the center of the structure. These electrons appear initially at rest and slip backwards relative to the bubble, then they are trapped and gain additional energy as they accelerate to the center of the accelerating structure. The only drawback of this method is that the electrons are continuously trapped into the bubble, which yields electrons with a large energy spread. Two-stage acceleration schemes, where electrons are trapped in a first stage containing a mix He/dopant and accelerated in a second stage containing pure helium ${ }^{25}$ can be implemented to obtain $\mathrm{GeV}$-class monoenergetic beams.

\subsection{Betatron x-ray radiation}

The motion of an electron accelerated along $\overrightarrow{u_{z}}$ with momentum $\vec{p}$ and position $\vec{r}$ in the wake of a laser pulse can be described by the Lorentz equation of motion:

$$
\frac{d \vec{p}}{d t}=-m \omega_{p}^{2} \frac{\vec{r}}{2}+\alpha \frac{m c \omega_{p}}{e} \overrightarrow{u_{z}}
$$

where $m$ is the electron rest mass, $e$ the elementary charge, and $\omega_{p}=\sqrt{n_{e} e^{2} / m \epsilon_{0}}$ is the plasma frequency. Here, $n_{e}$ is the electron density, and $\epsilon_{0}$ the vacuum permittivity. In the blowout $3 \mathrm{D}$ nonlinear regime of laser wakefield acceleration, ${ }^{20} \alpha=\frac{1}{2} \sqrt{a_{0}}$ is the normalized accelerating field, where $a_{0}$ is the laser normalized vector potential, typically around 2 for our experiments. Equation 1 can be solved by using a 4th order Runge-Kutta algorithm and obtain the single electron trajectories for given initial conditions and fields. The electron trajectory is used to calculate the intensity radiated by the particle per unit frequency $\omega$ and solid angle $\Omega:{ }^{26}$

$$
\frac{d^{2} I}{d \Omega d \omega}=\frac{e^{2} \omega^{2}}{4 \pi c}\left|\int_{-\infty}^{\infty} \vec{n} \times(\vec{n} \times \beta) e^{i \omega\left(t-\frac{\vec{n} \cdot \vec{r}}{c}\right)} d t\right|^{2},
$$



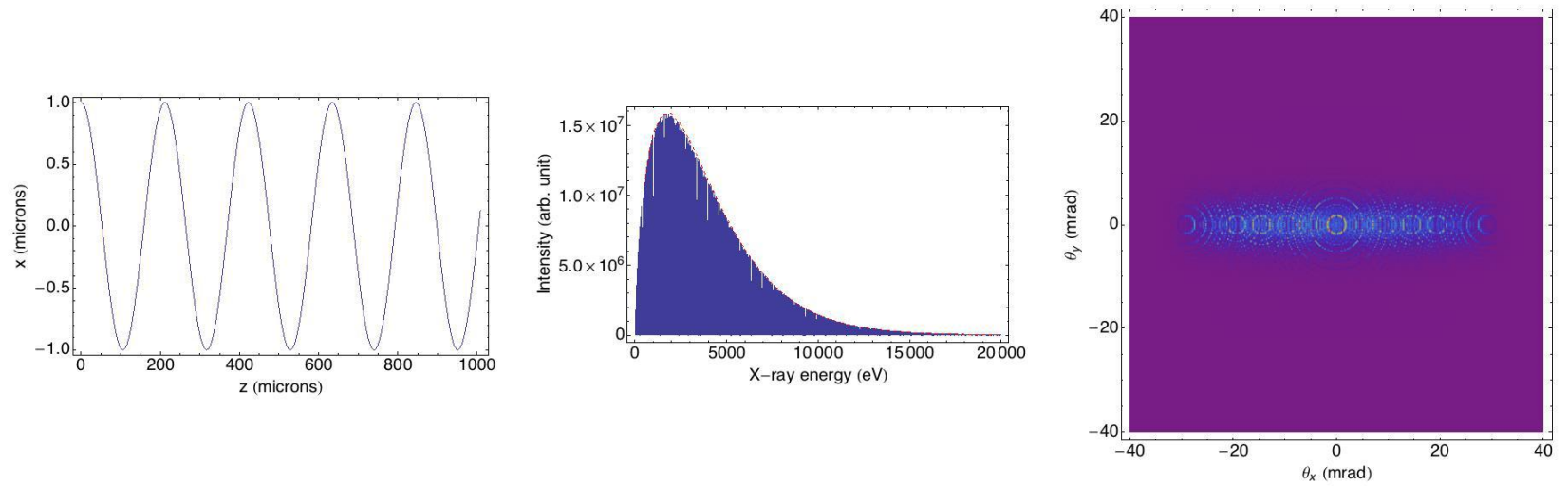

Figure 1. From left to right: example of an electron trajectory in the plasma, with the corresponding betatron spectrum observed on-axis and the x-ray beam profile. Shown on the center plot are the full spectrum (solid line), calculated using Equation 2 and containing the harmonic structure of the radiation, and the asymptotic limit (dashed red line), calculated using Equation 3. For this example, the parameters are $n_{e}=10^{19} \mathrm{~cm}^{-3}, \gamma=200, x_{0}=1 \mu \mathrm{m}, y_{0}=0$, and $\alpha=0$. Here the critical frequency $\hbar \omega_{c}[\mathrm{keV}]=5 \times 10^{-24} \times \gamma^{2} n_{e}\left[\mathrm{~cm}^{-3}\right] r[\mu \mathrm{m}]=4.2 \mathrm{keV}$ and $K \simeq 6$. The beam has a divergence of $1 / \gamma$ and $K / \gamma$ along the direction parallel and perpendicular to the plane of the oscillations.

where $\vec{n}$ is the vector corresponding to the direction of observation, and $\beta=v / c$ the normalized electron velocity. For relativistic energies, $\beta \sim 1$. In the case where the wiggler parameter $K=1.33 \times 10^{-10} \sqrt{\gamma n_{e}} r_{0}$ is larger than unity, the spectrum, observed at an angle $\theta$ from the plane in which the particle oscillates, can be approximated by the asymptotic limit: ${ }^{26,27}$

$$
\frac{d^{2} I}{d \Omega d \omega}=\frac{e^{2}}{3 \pi^{2} c}\left(\frac{\omega \rho}{c}\right)^{2}\left(\frac{1}{\gamma^{2}}+\theta^{2}\right)\left[K_{2 / 3}^{2}(\xi)+\frac{\theta^{2}}{\left(1 / \gamma^{2}\right)+\theta^{2}} K_{1 / 3}^{2}(\xi)\right],
$$

where $K_{2 / 3}$ and $K_{1 / 3}$ are modified Bessel functions. Here, $\rho$ is the radius of curvature of the electron trajectory and $\xi=\frac{\omega \rho}{3 c}\left(\frac{1}{\gamma^{2}}+\theta^{2}\right)^{3 / 2}$. When integrated over all angles of observation, the spectrum becomes:

$$
\frac{d I}{d \omega}=\sqrt{3} \frac{e^{2}}{c} \gamma \frac{\omega}{\omega_{c}} \int_{\frac{\omega}{\omega_{c}}}^{\infty} K_{5 / 3}(x) d x
$$

where $K_{5 / 3}$ is also a modified Bessel function and $\omega_{c}=3 \gamma^{3} c / \rho$ is the critical frequency. Equation 3 peaks at $\omega \sim 0.45 \omega_{c}$ for $\theta=0$ while Equation 4 peaks at $\omega \sim 0.3 \omega_{c}$. Similarly, the betatron x-ray beam profile is calculated by integrating Equation 2 over frequencies. Figure 1 shows an example of electron trajectory, with its corresponding betatron x-ray spectrum and beam profile. For this particular case, the parameters are $n_{e}=10^{19}$ $\mathrm{cm}^{-3}, \gamma=200, x_{0}=1 \mu \mathrm{m}, y_{0}=0$, and $\alpha=0$. The trajectory was calculated using 3000 time steps (with each unit step $\left.d t=0.2 c / \omega_{p}\right)$. At each point of calculation of the trajectory, the spectrum and beam profile were calculated using frequency steps of $100 \mathrm{eV}$. For the chosen parameters, $\omega_{c}=4.2 \mathrm{keV}$ and $K \simeq 6$. The beam has a divergence of $1 / \gamma$ and $K / \gamma$ along the direction parallel and perpendicular to the plane of the oscillations, and the on-axis spectrum peaks at $\sim 2 \mathrm{keV}$. Although more computationally intensive, electrons trajectories obtained from PIC simulations can be post-processed using Equation 2 to calculate the spectrum and profile with a much better resolution. ${ }^{28}$

\section{EXPERIMENTAL SETUP}

The experiments were conducted at the Jupiter Laser Facility (JLF) using the 200 TW Callisto laser system. Callisto can deliver up to $12 \mathrm{~J}$ in a $60 \mathrm{fs}$ pulse (full width at half maximum, fwhm). The $13 \mathrm{~cm}$ diameter beam was focused by an off-axis parabola ( $\mathrm{f} / 8$ or $\mathrm{f} / 20$ ) onto the $500 \mu \mathrm{m}$ entrance pinhole of a gas cell. Two different 
A

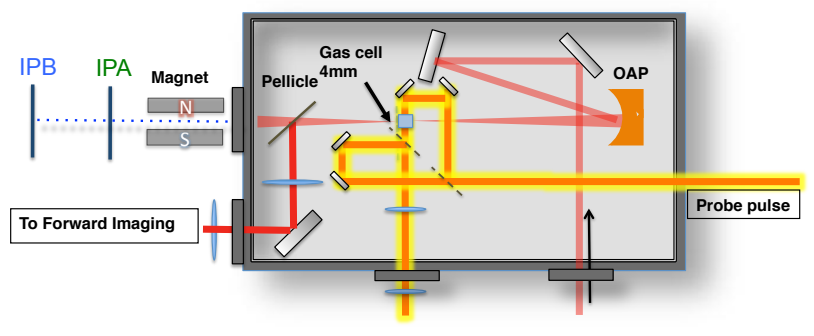

B

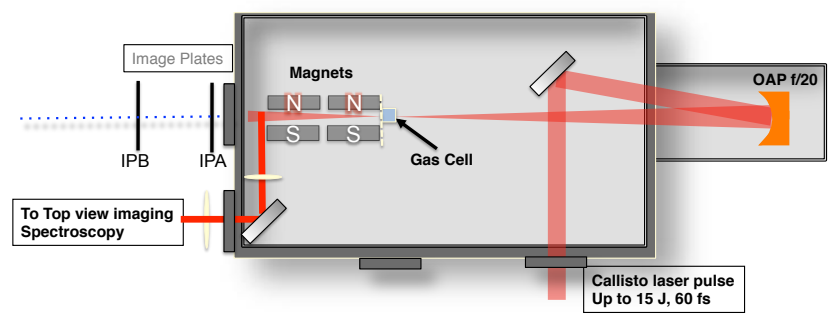

Figure 2. Experimental setups (A) and (B) for betatron x-rays production and detection (see text for details).

experimental setups, shown on Figure 2, were used. Provided that one can maintain $a_{0} \sim 2$ as an optimal value for the blowout regime, setup B should in principle be favorable for betatron x-rays due to the longer Rayleigh length and thus the ability for electrons to oscillate over a longer distance. The laser focal spot size $w_{0}$ was measured under vacuum at low power to be $12 \mu \mathrm{m}$ and $30 \mu \mathrm{m}$ (fwhm) for the $\mathrm{f} / 8$ and $\mathrm{f} / 20$ beam, respectively. The back pinhole is $1 \mathrm{~mm}$ wide, and we have used different gas cell lengths (from $3 \mathrm{~mm}$ to $10 \mathrm{~mm}$ ) as well as two-stage designs. ${ }^{25}$ The cell was typically filled with He gas, but also with N, CO2, Ar and Ne. Electron densities ranging from $2 \times 10^{18} \mathrm{~cm}^{-3}$ to $1.5 \times 10^{19} \mathrm{~cm}^{-3}$ were measured for each shot with a 100 fs probe using interferometry in combination with an Abel-inversion code. The laser spot at the exit of the gas cell was imaged using a forward diagnostic, and spectrally resolved using grating and prism-based spectrometers. The electron beams were characterized using a two-screen spectrometer. ${ }^{24,29,30}$ This system provides an accurate measurement of the energy of the electrons, the vertical and horizontal angle that the electrons exit the plasma relative to the original laser axis, the divergence, and the electron charge. The electrons exiting the plasma are deflected in the vertical direction by a 21 -cm-long, 0.42 Tesla dipole magnet (setup A). The electrons are detected by two successive image plates $\left(\mathrm{IP}_{a}\right.$ and $\left.\mathrm{IP}_{b}\right)$ allowing for a unique solution to their energy and deflection, provided common spatial features can be identified on both image plates. In the case of setup B, we used two magnets instead of one, and they were moved closer to the interaction region (in the vacuum chamber) in order to produce a larger deflection of the electron beam (with respect to the x-ray beam) on the image plates. For this second setup the image plates were also moved closer to the target chamber in order to minimize the x-ray propagation through air. Both the betatron x-ray beam profile and the x-ray spectrum were measured on the first image plate $\mathrm{IP}_{a}$. At the vacuum/air interface, we used a $12 \mu \mathrm{m}$ thick $\mathrm{Al}$ foil to block the $800 \mathrm{~nm}$ light from the laser as well as a $60 \mu \mathrm{m}$ mylar window to hold vacuum. X-rays (profile or spectrum) are then measured on the image plate. To measure the x-ray spectrum from $1 \mathrm{keV}$ to $30 \mathrm{keV}$, we used a set of Ross filter pairs, with transmission functions that provide narrow energy bands. The number of x-ray photons per unit energy is given by $N_{p h}=S_{n} /\left(T_{n} \Delta E_{n}\right)$, where $S_{n}, T_{n}$ and $\Delta E_{n}$ are respectively the signal, transmission and bandwidth of a given energy band. Shown on Figure 3 is a typical x-ray beam observed on $\mathrm{IP}_{a}$ through the pie-shaped sets of filters, as well as the energy bands used in the experiment.

\section{RESULTS AND DISCUSSION}

\subsection{Observation of betatron x-rays in different gases}

Betatron x-rays where observed in different gases. For the different profiles shown on Figure 4, observed on $\mathrm{IP}_{a}$ through the filters, the x-rays where produced in a $4 \mathrm{~mm}$ gas cell containing mixed gases. Although these $\mathrm{x}$-rays where produced in different gases, in order to make quantitative arguments it is important to note that they correspond to different electron beam energies. The electron beam maximum energy (the largest contribution to the x-rays) is, for each of these shots: $234 \mathrm{MeV}, 300 \mathrm{MeV}, 385 \mathrm{MeV}, 186 \mathrm{MeV}, 280 \mathrm{MeV}, 125 \mathrm{MeV}$ (from top left, clockwise). Electron densities were in the $5 \times 10^{18}-1 \times 10^{19} \mathrm{~cm}^{-3}$ range for each of these shots and $2<a_{0}<2.5$. In some cases (Figure 4) the beam profile could be very well observed on both $\mathrm{IP}_{a}$ and $\mathrm{IP}_{b}$ with a $25 \%$ transmission from $\mathrm{IP}_{a}$ to $\mathrm{IP}_{b}$. According to the theoretical and experimental response function and transmission curve of the image plates, ${ }^{31}$ it means that the beam contains a large amount of x-rays with energies above $20 \mathrm{keV}$. For this particular case, the electron beam peaked at $\sim 385 \mathrm{MeV}$, where most of the charge was located. Since the critical 

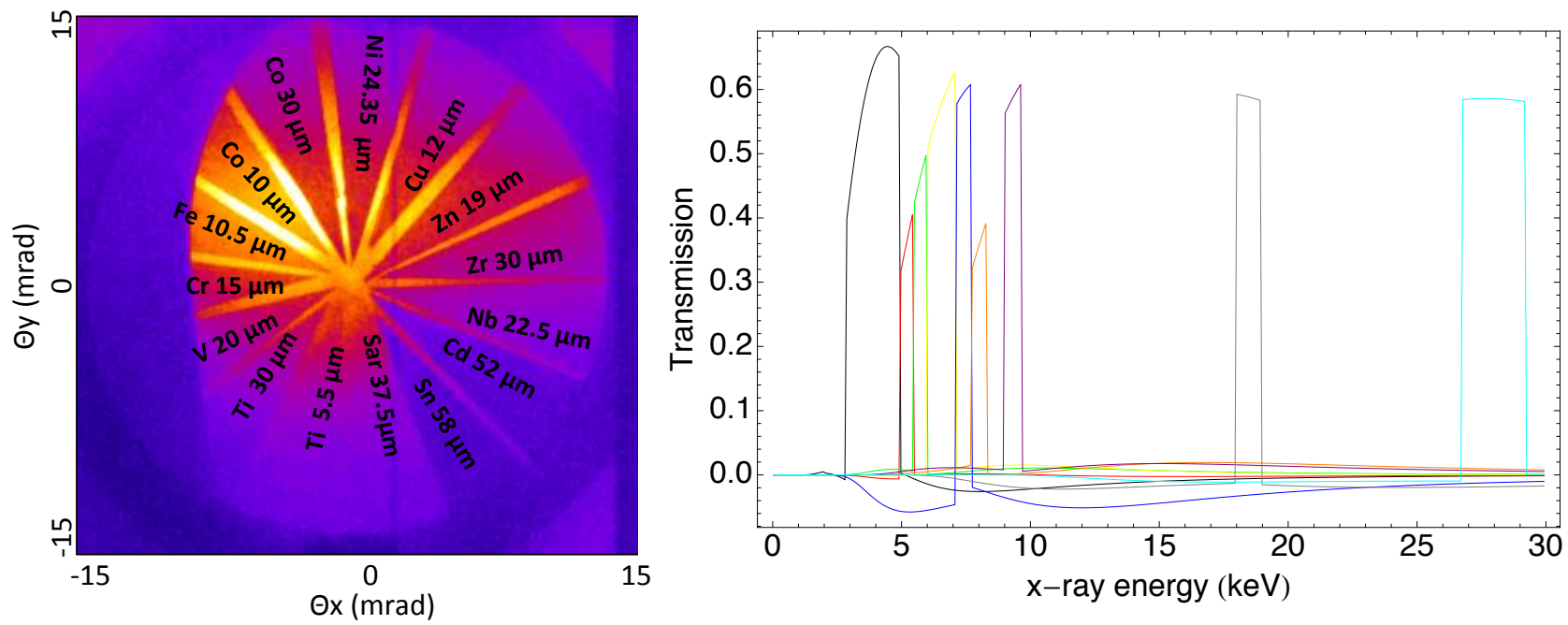

Figure 3. Betatron x-ray beam observed on $\mathrm{IP}_{a}$ through the pie-shaped set of filters (left) and corresponding Ross filter pairs used to measure the spectrum (right). Each pair corresponds to the difference of signal observed through two specific filters. The nine pairs (and their respective mean energy $E_{n}$ and bandwidth $\Delta E_{n}$ ) are, from low to high x-ray energy: $5.5 \mu \mathrm{m}$ Ti and $37.5 \mu \mathrm{m}$ Saran $(3.9 \mathrm{keV}, 2 \mathrm{keV}), 20 \mu \mathrm{m} \mathrm{V}$ and $30 \mu \mathrm{m}$ Ti $(5.2 \mathrm{keV}, 0.45 \mathrm{keV}), 15 \mu \mathrm{m} \mathrm{Cr}$ and $20 \mu \mathrm{m} \mathrm{V}(5.7$ $\mathrm{keV}, 0.45 \mathrm{keV}), 10.5 \mu \mathrm{m}$ Fe and $15 \mu \mathrm{m} \mathrm{Cr}(6.5 \mathrm{keV}, 1.05 \mathrm{keV}), 10 \mu \mathrm{m}$ Co and $15 \mu \mathrm{m} \mathrm{Fe}(7.4 \mathrm{keV}, 0.53 \mathrm{keV}), 24.35 \mu \mathrm{m}$ $\mathrm{Ni}$ and $30 \mu \mathrm{m}$ Co $(8.3 \mathrm{keV}, 0.52 \mathrm{keV}), 19 \mu \mathrm{m} \mathrm{Zn}$ and $17.5 \mu \mathrm{m} \mathrm{Cu}(9.3 \mathrm{keV}, 0.6 \mathrm{keV}), 22.5 \mu \mathrm{m} \mathrm{Nb}$ and $30 \mu \mathrm{m} \mathrm{Zn}(18.5$ $\mathrm{keV}, 0.9 \mathrm{keV}), 58 \mu \mathrm{m}$ Sn and $52 \mu \mathrm{m} \mathrm{Cd}(28 \mathrm{keV}, 2.3 \mathrm{keV})$.

frequency scales with $\gamma^{2}$ and that in practical units, $\hbar \omega_{c}[\mathrm{keV}]=5 \times 10^{-24} \times \gamma^{2} n_{e}\left[\mathrm{~cm}^{-3}\right] r[\mu \mathrm{m}]$, it means that for $n_{e}=10^{19} \mathrm{~cm}^{-3}$ and $\gamma=750, r \simeq 0.7 \mu \mathrm{m}$ when the electrons reach their end energy. The drawback of using filter wedges in our experiments was the beam pointing jitter. As observed on Figure 4, the beam center location varies up to a few milliradians around the filter tips. Since the x-ray intensity and energy strongly depend on the angle of observation (Equation 2), making quantitative statements about the x-ray spectrum requires x-ray measurements be made at the same angular position with respect to the beam center.

\subsection{Observation of electron spectral oscillations}

In a second series of experiments, we used our recently developed two-stage gas cell, ${ }^{25}$ in conjunction with setup $\mathrm{B}$, to produce and observe betatron $\mathrm{x}$-rays. This setup is more advantageous for $\mathrm{x}$-ray detection because the x-ray absorption in air ( respectively $50 \%$ and $80 \%$ transmission at $5 \mathrm{keV}$ for $70 \mathrm{~cm}$ and $5 \mathrm{~cm}$ ) is minimized, and the beam profile containing even the lowest energies can be observed. In some cases, we simultaneously observed the betatron beam profile and spectral oscillations of the electrons. For the example shown on Figure 5, the laser energy was $6.7 \mathrm{~J}$, while the other parameters are identical to Section 3. The target was a two-stage gas cell with a $2.5 \mathrm{~mm}$ injector containing $25 \%$ of $\mathrm{N}$ and $75 \%$ of $\mathrm{He}$, and a $13.75 \mathrm{~mm}$ accelerator filled with $100 \% \mathrm{He}$. The electron density, measured with interferometry, was $2 \times 10^{18} \mathrm{~cm}^{-3}$. At such a low density, and for our laser parameters, electrons oscillating in the plasma cannot be self-trapped from helium, and are primarily from the outer-shell nitrogen electrons. Indeed, in this case, the laser peak power was $122 \mathrm{TW}$, with a $30 \%$ coupling into the wake. For an electron density of $n_{e}=2 \times 10^{18} \mathrm{~cm}^{-3}$, it means $P / P_{\text {crit }} \sim 2.5$, where $P_{\text {crit }}=17 \omega_{0}^{2} / \omega_{p}^{2}[\mathrm{GW}]$ is the critical power for relativistic focusing. According to experiments performed at Callisto, self-trapping does not occur if $P / P_{\text {crit }}<3 .{ }^{32}$ On Figure 5, the electron oscillations can be very well reproduced by solving the equation of motion using the Runge-Kutta method as described in Section 2. For this example $n_{e}=2 \times 10^{18}$ $\mathrm{cm}^{-3}, \gamma_{0}=5, \alpha=0.85$, and $x_{0}=0.3 \mu \mathrm{m}$. The electrons oscillate primarily along the laser polarization axis, as it has also been observed in prior betatron x-rays related experiments. ${ }^{13,14}$ 

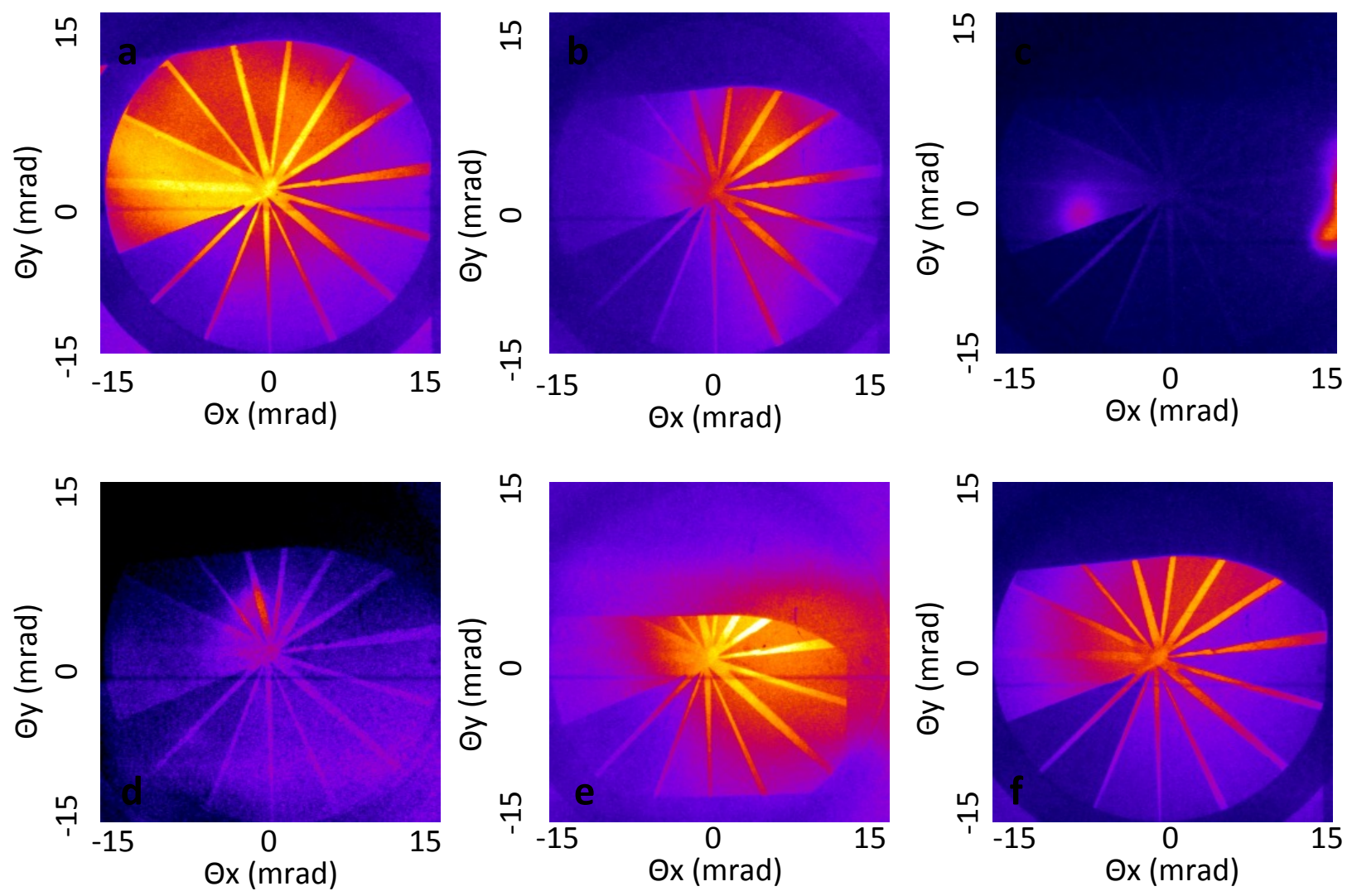

Figure 4. Betatron x-ray beam beam profiles observed on $\mathrm{IP}_{a}$ through the pie-shaped set of filters (see text for details). The gas composition for each shot was (from top left, clockwise): 100\% He, 95\% He and 5\% Ar, 80\% He and 20\% N2, $80 \%$ He and $20 \%$ N2, $98 \%$ He and $2 \%$ Ar, $95 \%$ Ar and $5 \%$ He.
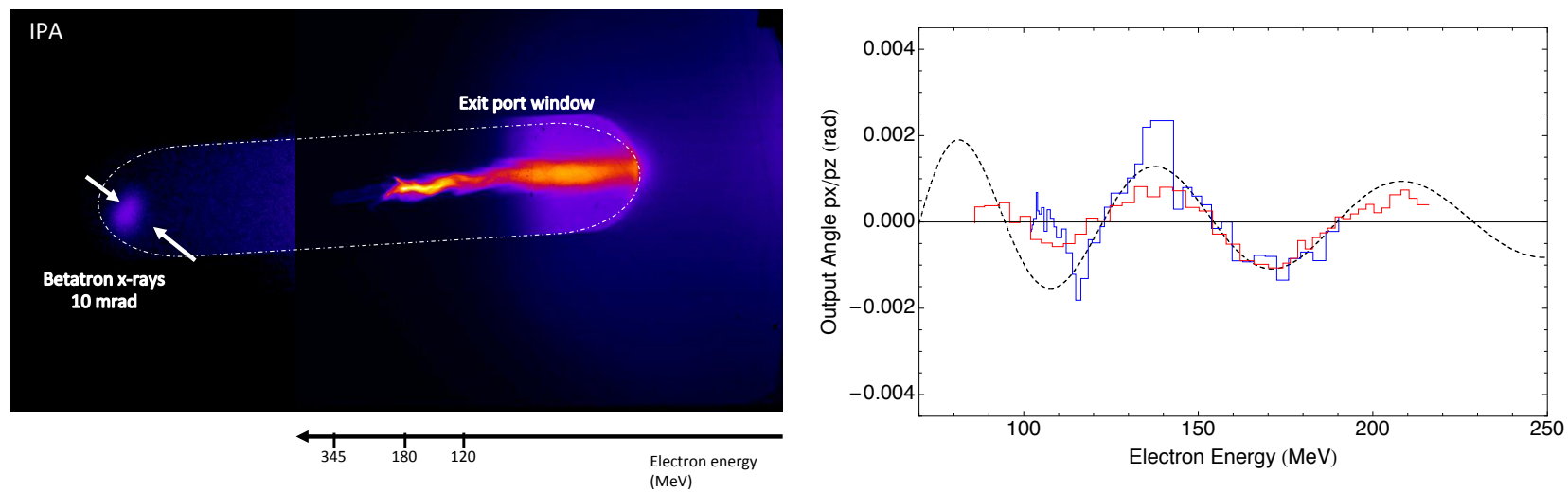

Figure 5. Electron beam spectrum and betatron x-ray beam profile observed on $\mathrm{IP}_{a}$ (left) and corresponding output angle vs. energy plot (right). The solid curves correspond to the data measured on the image plates and the dashed line is the trajectory calculated by solving Equation 1 with the parameters: $n_{e}=2 \times 10^{18} \mathrm{~cm}^{-3}, \gamma_{0}=5, \alpha=0.85, x_{0}=0.3 \mu \mathrm{m}$, $y_{0}=0$. The laser is polarized along the smaller dimension of the image plate. The contrast has been artificially enhanced on the left side of the picture to show the betatron beam profile. 


\section{CONCLUSION AND PERSPECTIVES}

In conclusion we have observed betatron x-rays at energies beyond $20 \mathrm{keV}$ in a collimated beam with $<30 \mathrm{mrad}$ divergence. X-rays have been observed in pure $\mathrm{He}$, but also in $\mathrm{He} / \mathrm{N}$ and $\mathrm{He} / \mathrm{Ar}$ mixes, which opens several possibilities to improve and control the x-ray flux and spectrum of the source. By taking advantage of electron acceleration in the ionization-induced trapping regime, large amounts of charge can be trapped and produce bright betatron x-rays. Our preliminary measurements support betatron oscillations of $\sim 1 \mu \mathrm{m}$ in the plasma, but also show that a simultaneous measurement of the x-ray beam profile and spectrum at a particular angle is necessary in order to make quantitative arguments about betatron x-rays production in this regime.

\section{ACKNOWLEDGMENTS}

This work was performed under the auspices of the U.S. Department of Energy under contract DE-AC5207NA27344, and supported by the Laboratory Directed Research and Development (LDRD) Program under tracking code 13-LW-076. The authors thank R.C. Cauble, J. Bonlie and S. Maricle for their support of the Callisto laser system at the Jupiter Laser Facility, and F.A. acknowledges fruitful discussions with F.V. Hartemann on theory and modeling.

\section{REFERENCES}

1. T.Tajima and J.M. Dawson, "Laser electron accelerator," Phys. Rev. Lett. 43, 267-270 (1979).

2. S. P. D. Mangles, C. D. Murphy, Z. Najmudin, A. G. R. Thomas, J. L. Collier, A. E. Dangor, E. J. Divall, P. S. Foster, J. G. Gallacher, C. J. Hooker, D. A. Jaroszynski, A. J. Langley, W. B. Mori, P. A. Norreys, F. S. Tsung, R. Viskup, B. R. Walton and K. Krushelnick,, "Monoenergetic beams of relativistic electrons from intense laser-plasma interactions," Nature 431, 535-538 (2004).

3. C. G. R. Geddes, Cs. Toth, J. van Tilborg, E. Esarey, C. B. Schroeder, D. Bruhwiler, C. Nieter, J. Cary and W. P. Leemans, "High-quality electron beams from a laser wakefield accelerator using plasma-channel guiding," Nature 431, 538-541 (2004).

4. J. Faure, Y. Glinec, A. Pukhov, S. Kiselev, S. Gordienko, E. Lefebvre, J.-P. Rousseau, F. Burgy and V. Malka, "A laser-plasma accelerator producing monoenergetic electron beams," Nature 431, 541-544 (2004).

5. P. Emma, R. Akre, J. Arthur, R. Bionta, C. Bostedt, J. Bozek, A. Brachmann, P. Bucksbaum, R. Coffee, F.-J. Decker, Y. Ding, D. Dowell, S. Edstrom, A. Fisher, J. Frisch, S. Gilevich, J. Hastings, G. Hays, Ph. Hering, Z. Huang, R. Iverson, H. Loos, M. Messerschmidt, A. Miahnahri, S. Moeller, H.-D. Nuhn, G. Pile, D. Ratner, J. Rzepiela, D. Schultz, T. Smith, P. Stefan, H. Tompkins, J. Turner, J. Welch, W. White, J. Wu, G. Yocky and J. Galayda, "First lasing and operation of an angstrom-wavelength free-electron laser," Nat. Photon. 4, 641-647 (2010).

6. Matthias Fuchs, Raphael Weingartner, Antonia Popp, Zsuzsanna Major, Stefan Becker, Jens Osterhoff, Isabella Cortrie, Benno Zeitler, Rainer Horlein, George D. Tsakiris, Ulrich Schramm, Tom P. Rowlands-Rees, Simon M. Hooker, Dietrich Habs, Ferenc Krausz, Stefan Karsch Florian Gruner, "Laser-driven soft-x-ray undulator source," Nat. Phys. 5, 826-829 (2009).

7. Antoine Rousse, Kim Ta Phuoc, Rahul Shah, Alexander Pukhov, Eric Lefebvre, Victor Malka, Sergey Kiselev, Frederic Burgy, Jean-Philippe Rousseau, Donald Umstadter, and Daniele Hulin, "Production of a kev x-ray beam from synchrotron radiation in relativistic laser-plasma interaction," Phys. Rev. Lett. 93, 135005 (2004).

8. S. Corde, K. Ta Phuoc, G. Lambert, R. Fitour, V. Malka, and A. Rousse, "Femtosecond x rays from laserplasma accelerators," Rev. Mod. Phys. 85, 1-47 (2013).

9. Shuoqin Wang, C. E. Clayton, B. E. Blue, E. S. Dodd, K. A. Marsh, W. B. Mori, C. Joshi, S. Lee, P. Muggli, T. Katsouleas, F. J. Decker, M. J. Hogan, R. H. Iverson, P. Raimondi, D. Walz, R. Siemann, and R. Assmann, "X-ray emission from betatron motion in a plasma wiggler," Phys. Rev. Lett. 88, 135004 (2002).

10. Felicie Albert, Rahul Shah, Kim Ta Phuoc, Romuald Fitour, Frederic Burgy, Jean-Philippe Rousseau, Amar Tafzi, Denis Douillet, Thierry Lefrou, and Antoine Rousse, "Betatron oscillations of electrons accelerated in laser wakefields characterized by spectral x-ray analysis," Phys. Rev. E 77, 056402 (2008). 
11. S. Fourmaux, S. Corde, K. Ta Phuoc, P. M. Leguay, S. Payeur, P. Lassonde, S. Gnedyuk, G. Lebrun, C. Fourment, V. Malka, S. Sebban, A. Rousse and J. C. Kieffer, "Demonstration of the synchrotron-type spectrum of laser-produced betatron radiation," New J. Phys. 13, 033017 (2011).

12. R. C. Shah, F. Albert, K. Ta Phuoc, O. Shevchenko, D. Boschetto, A. Pukhov, S. Kiselev, F. Burgy, J.P. Rousseau, and A. Rousse, "Coherence-based transverse measurement of synchrotron x-ray radiation from relativistic laser-plasma interaction and laser-accelerated electrons," Phys. Rev. E 74, 045401(R) (2006).

13. S. Kneip, C. McGuffey, J. L. Martins, S. F. Martins, C. Bellei, V. Chvykov, F. Dollar, R. Fonseca, C. Huntington, G. Kalintchenko, A. Maksimchuk, S. P. D. Mangles, T. Matsuoka, S. R. Nagel, C. A. J. Palmer, J. Schreiber, K. Ta Phuoc, A. G. R. Thomas, V. Yanovsky, L. O. Silva, K. Krushelnick and Z. Najmudin, "Bright spatially coherent synchrotron x-rays from a table-top source," Nat. Phys. 6, 980-983 (2010).

14. Kim Ta Phuoc, Sebastien Corde, Rahul Shah, Felicie Albert, Romuald Fitour, Jean-Philippe Rousseau, Frederic Burgy, Brigitte Mercier, and Antoine Rousse, "Imaging electron trajectories in a laser-wakefield cavity using betatron x-ray radiation," Phys. Rev, Lett 97, 225002 (2006).

15. K. Ta Phuoc, R. Fitour, A. Tafzi, T. Garl, N. Artemiev, R. Shah, F. Albert, D. Boschetto, A. Rousse, D-E. Kim, A. Pukhov, V. Seredov, and I. Kostyukov, "Demonstration of the ultrafast nature of laser produced betatron radiation," Phys. Plasmas 14, 080701 (2007).

16. S. Kneip, C. McGuffey, F. Dollar, M. S. Bloom, V. Chvykov, G. Kalintchenko, K. Krushelnick, A. Maksimchuk, S. P. D. Mangles, T. Matsuoka, Z. Najmudin, C. A. J. Palmer, J. Schreiber, W. Schumaker, A. G. R. Thomas, and V. Yanovsky, "X-ray phase contrast imaging of biological specimens with femtosecond pulses of betatron radiation from a compact laser plasma wakefield accelerator," Appl. Phys. Lett. 99, 093701 (2011).

17. S. Fourmaux, S. Corde, K. Ta Phuoc, P. Lassonde, G. Lebrun, S. Payeur, F. Martin, S. Sebban, V. Malka, A. Rousse, and J. C. Kieffer, "Single shot phase contrast imaging using laser-produced betatron x-ray beams," Opt. Lett. 36, 2426-2428 (2011).

18. G. R. Plateau, C. G. R. Geddes, D. B. Thorn, M. Chen, C. Benedetti, E. Esarey, A. J. Gonsalves, N. H. Matlis, K. Nakamura, C. B. Schroeder, S. Shiraishi, T. Sokollik, J. van Tilborg, Cs. Toth, S. Trotsenko, T. S. Kim, M. Battaglia, Th. Stohlker, and W. P. Leemans, "Low-emittance electron bunches from a laser-plasma accelerator measured using single-shot x-ray spectroscopy," Phys. Rev. Lett. 109, 064802 (2012).

19. S. Kneip, C. McGuffey, J. L. Martins, M. S. Bloom, V. Chvykov, F. Dollar, R. Fonseca, S. Jolly, G. Kalintchenko, K. Krushelnick, A. Maksimchuk, S. P. D. Mangles, Z. Najmudin, C. A. J. Palmer, K. Ta Phuoc, W. Schumaker, L. O. Silva, J. Vieira, V. Yanovsky, and A. G. R. Thomas, "Characterization of transverse beam emittance of electrons from a laser-plasma wakefield accelerator in the bubble regime using betatron x-ray radiation," Phys. Rev. ST Accel. Beams 15, 021302 (2012).

20. W. Lu, M. Tzoufras, C, Joshi, F.S. Tsung, W.B. Mori, J. Vieira, R. A. Fonseca and L.O. Silva, "Generating multi-gev electron bunches using single stage laser wakefield acceleration in a $3 \mathrm{~d}$ nonlinear regime," Phys. Rev. ST Acc. Beams 10, 061301 (2007).

21. A. Pak, K. A. Marsh, S. F. Martins, W. Lu, W. B. Mori, and C. Joshi, "Injection and trapping of tunnelionized electrons into laser-produced wakes," Phys. Rev. Lett 104, 025003 (2010).

22. C. McGuffey, A. G. R. Thomas, W. Schumaker, T. Matsuoka, V. Chvykov, F. J. Dollar, G. Kalintchenko, V. Yanovsky, A. Maksimchuk, and K. Krushelnick, "Ionization induced trapping in a laser wakefield accelerator," Phys. Rev. Lett. 104, 025004 (2010).

23. W. P. Leemans, B. Nagler, A. Gonzalves, C. Toth, K. Nakamura, C. Geddes, E. Esarey, E. Schroeder and S. Hooker, "Gev electron beams from a centimetre-scale accelerator," Nat. Phys. 2, 696 (2006).

24. C. E. Clayton, J. E. Ralph, F. Albert, R. A. Fonseca, S. H. Glenzer, C. Joshi, W. Lu, K. A. Marsh, S. F. Martins, W. B. Mori, A. Pak, F. S. Tsung, B. B. Pollock, J. S. Ross, L. O. Silva, and D. H. Froula, "Selfguided laser wakefield acceleration beyond 1 gev using ionization-induced injection," Phys. Rev. Lett 105, 105003 (2010).

25. B. B. Pollock, C. E. Clayton, J. E. Ralph, F. Albert, A. Davidson, L. Divol, C. Filip, S. H. Glenzer, K. Herpoldt, W. Lu, K. A. Marsh, J. Meinecke, W. B. Mori, A. Pak, T. C. Rensink, J. S. Ross, J. Shaw, G. R. Tynan, C. Joshi,and D. H. Froula, "Demonstration of a narrow energy spread, 0,5 gev electron beam from a two-stage laser wakefield accelerator," Phys. Rev. Lett 107, 045001 (2011).

26. J.D. Jackson, [Classical Electrodynamics] (1998). 
27. E. Esarey, B. A. Shadwick, P. Catravas, and W. P. Leemans, "Synchrotron radiation from electron beams in plasma-focusing channels," Phys. Rev. E 65, 056505 (2002).

28. J.L. Martins, S.F. Martins, R.A. Fonseca and L.O. Silva, Proc. SPIE 7359, 73590V-1-73590V-8 (2009).

29. Ian Blumenfeld, Christopher E. Clayton, Franz-Josef Decker, Mark J. Hogan, Chengkun Huang, Rasmus Ischebeck, Richard Iverson, Chandrashekhar Joshi, Thomas Katsouleas, Neil Kirby, Wei Lu, Kenneth A. Marsh, Warren B. Mori, Patric Muggli, Erdem Oz, Robert H. Siemann, Dieter Walz and Miaomiao Zhou, "Energy doubling of $42 \mathrm{gev}$ electrons in a metre-scale plasma wakefield accelerator," Nature 445, 741-744 (2006).

30. B. B. Pollock, J.S. Ross, G. R. Tynan, L. Divol, S. H. Glenzer, V. Leurent, J. P. Palastro, J.E. Ralph, D.H. Froula, C. E. Clayton, K.A. Marsh, A. E. Pak, T.L. Wang, C. Joshi, "Two-screen method for determining electron beam energy and deflection from laser wakefield acceleration," Proceedings of PAC09, Vancouver, BC Canada, 3035-3037 (2009).

31. B. R. Maddox, H. S. Park, B. A. Remington, N. Izumi, S. Chen, C. Chen, G. Kimminau, Z. Ali, M. J. Haugh, and Q. Ma, "High-energy x-ray backlighter spectrum measurements using calibrated image plates," Rev. Sc. Instr. 82, 023111 (2011).

32. D. H. Froula, C. E. Clayton, T. Doppner, K. A. Marsh, C. P. J. Barty, L. Divol, R. A. Fonseca, S. H. Glenzer, C. Joshi, W. Lu, S. F. Martins, P. Michel, W. B. Mori, J. P. Palastro, B. B. Pollock, A. Pak, J. E. Ralph, J. S. Ross, C. W. Siders, L. O. Silva, and T. Wang, "Measurements of the critical power for self-injection of electrons in a laser wakefield accelerator," Phys. Rev. Lett. 103, 215006 (2009). 